\title{
Soft gluon resummation for slepton pair-production
}

\author{
Alessandro Broggio* \\ Institut für Physik (THEP), \\ Johannes Gutenberg-Universität, D-55099 Mainz, Germany \\ E-mail: broggio@uni-mainz.de
}

Matthias Neubert

Institut für Physik (THEP),

Johannes Gutenberg-Universität, D-55099 Mainz, Germany

E-mail: matthias.neubert@uni-mainz.de

\section{Leonardo Vernazza ${ }^{\dagger}$}

Institut für Physik (THEP),

Johannes Gutenberg-Universität, D-55099 Mainz, Germany

E-mail: vernazza@uni-mainz.de

We report on recent results on the differential cross section for slepton pair-production at hadron colliders. We use an approach to threshold resummation, based on soft-collinear effective theory, to quantify the dynamical enhancement of the partonic threshold region. We evaluate the resummed invariant mass distribution and total cross section at the next-to-next-to-next-to-leading logarithmic order, and match the result onto next-to-leading order calculation.

The 2011 Europhysics Conference on High Energy Physics-HEP 2011,

July 21-27, 2011

Grenoble, Rhône-Alpes, France

*Speaker.

${ }^{\dagger}$ Alexander-von-Humboldt Fellow 


\section{Introduction}

With the advent of the Large Hadron Collider (LHC), the experimental investigation of TeVscale physics is now fully accessible. The stabilization of the electroweak scale requires new particles and interactions in the $\mathrm{TeV}$ range, and supersymmetry is one of the most compelling scenario which achieves such a stabilization. It predicts the existence of "supersymmetric partners" for all of the SM particles. The superpartners are expected to have masses of the order of a TeV.

The scalar-leptons are expected to be among the lightest supersymmetric particles and, in many scenarios, they decay directly into their corresponding SM partners and the lightest supersymmetric particle. The production of a slepton-pair should have quite simple signatures like a couple of energetic leptons plus missing energy.

Currently, complete next-to-leading order (NLO) calculations are available in SUSY-QCD for the total slepton-pair production cross section [1], as well for the differential distributions [2]. Reducing the scale uncertainty by computing higher order terms in the perturbative expansion is a task which becomes soon prohibitive due to the number of different scales which enter the problem. A good alternative to improve the theoretical prediction is to resort to threshold resummation methods [3], which allows one to take into account the dominant contributions of the higher order terms. Expressions for the resummed invariant mass distribution and total cross section were obtained in Mellin moment space at the next-to-leading logarithmic accuracy in [2]. We extend this result to next-to-next-to-next-to-leading logarithmic (NNNLL) accuracy using soft-collinear effective theory tecniques. In this work we summarize the results reported in [3].

\section{Differential cross section}

We consider the process

$$
N_{1}\left(P_{1}\right)+N_{2}\left(P_{2}\right) \rightarrow \gamma^{*}, Z^{0 *}+X \rightarrow \tilde{l}\left(p_{3}\right), \tilde{l}^{*}\left(p_{4}\right)+X,
$$

where $N_{1}, N_{2}$ indicate two protons (LHC case) and $X$ represents an inclusive hadronic final state. The doubly-differential cross section in the invariant mass $M^{2}$ and in the rapidity $Y$ for the process in Eq. (2.1) can be written as

$$
\frac{d^{2} \sigma}{d M^{2} d Y}=\frac{\pi \alpha_{\mathrm{em}} \beta_{\tilde{l}}^{3}}{3 N_{c} M^{2} s} \sum_{i j} \int d x_{1} d x_{2} \tilde{C}_{i j}\left(x_{1}, x_{2}, \hat{s}, M, m_{\tilde{q}}, m_{\tilde{g}}, \mu_{f}\right) f_{i / N_{1}}\left(x_{1}, \mu_{f}\right) f_{j / N_{2}}\left(x_{2}, \mu_{f}\right),
$$

where $f_{i / N}$ are the parton distribution functions, $\tilde{C}_{i j}$ are the hard-scattering kernels, $s(\hat{s})$ is the hadronic (partonic) center of mass energy squared and $\beta_{\tilde{l}}=\sqrt{1-4 m_{\tilde{l}}^{2} / M^{2}}$. At NLO the sum extends over the following channels: $(i j)=(q \bar{q}),(\bar{q} q),(q g),(g q),(\bar{q} g),(g \bar{q})$. We are interested in the evaluation of the SUSY-QCD corrections near threshold, for this pourpose it is useful to introduce the following quantities:

$$
\tau=\frac{M^{2}}{s}, \quad z=\frac{M^{2}}{\hat{s}}=\frac{\tau}{x_{1} x_{2}},
$$

where $\hat{s}=x_{1} x_{2} s$. We define the partonic threshold as the region of the phase space where $\hat{s} \rightarrow M^{2}$ $(z \rightarrow 1)$. In this regime the dynamics of the process is greatly simplified: the partonic center-ofmass energy $\sqrt{\hat{s}}$ is just enough to create a slepton-pair, and there is no phase space available for 
the emission of hard gluons or soft quarks. The cross section is dominated by the terms which are singular in the $z \rightarrow 1$ limit, which correspond to the virtual corrections and the real emission of soft gluons. After integrating over the rapidity and retaining only the contributions which are singular in the threshold region, it is possible to write the invariant mass distribution of the process in Eq. (2.1) as

$$
\frac{d \sigma^{\text {thresh }}}{d M^{2}}=\frac{\pi \alpha_{\mathrm{em}} \beta_{\tilde{l}}^{3}}{3 N_{c} M^{2} s} \sum_{q} f_{q}^{\tilde{l}} \int_{\tau}^{1} \frac{d z}{z} C\left(z, M, m_{\tilde{q}}, m_{\tilde{g}}, \mu_{f}\right) f f\left(\tau / z, \mu_{f}\right)
$$

where

$$
f_{q}^{\tilde{l}}=\left[e_{q}^{2}-\frac{e_{q}\left(g_{L}^{q}+g_{R}^{q}\right) g^{\tilde{l}, Z}}{1-m_{Z}^{2} / M^{2}}+\frac{1}{2} \frac{\left(g_{L}^{q 2}+g_{R}^{q 2}\right) g^{\tilde{l}, Z^{2}}}{\left(1-m_{Z}^{2} / M^{2}\right)^{2}}\right],
$$

and

$$
f f\left(y, \mu_{f}\right)=\int_{y}^{1} \frac{d x}{x}\left[f_{q / N_{1}}\left(x, \mu_{f}\right) f_{\bar{q} / N_{2}}\left(y / x, \mu_{f}\right)+(q \leftrightarrow \bar{q})\right],
$$

defines the parton luminosity function. The hard-scattering kernel $C\left(z, M, m_{\tilde{q}}, m_{\tilde{g}}, \mu_{f}\right)$ depends on the invariant mass $M$, on the ratio $z$, as well on the masses of squarks and gluinos, $m_{\tilde{q}}, m_{\tilde{g}}$. By using soft-collinear effective theory methods, it is possible to prove that in the $z \rightarrow 1$ limit the hard-scattering kernel factorize in the following way:

$$
C\left(z, M, m_{\tilde{q}}, m_{\tilde{g}}, \mu_{f}\right)=H\left(M, m_{\tilde{q}}, m_{\tilde{g}}, \mu_{f}\right) S\left(\sqrt{\hat{s}}(1-z), \mu_{f}\right) .
$$

The hard function $H$ is related to virtual corrections, while the soft function $S$ originate from the real emission of soft gluons. At $n$-th order in perturbation theory the soft function involve plus distributions of the form $\alpha_{s}^{n}\left[\ln ^{m}(1-z) /(1-z)\right]_{+}$, where $m=0, \ldots, 2 n-1$, which need to be resummed to all orders. The resummation of these singular threshold logarithms can be accomplished by solving renormalization-group equations for the hard and the soft function in the effective theory. To obtain the best possible predictions we perform the resummation at NNNLL accuracy and then match the result onto NLO calculation.

\section{Phenomenology}

In this section we show some numerical results for the resummed invariant mass distribution and the total cross section for the LHC at $7 \mathrm{TeV}$ center-of-mass energy. For a detailed discussion we refer to [3]. The results for the invariant mass distribution shown in Fig. (1) have been obtained by using MSTW2008 PDFs [4]. We consider the PDFs at the order which is appropriate for the expansion in perturbation theory of the corresponding hard-scattering kernel, i.e., we consider LO and NLO PDFs for the full LO and NLO distributions, NLO and NNLO PDFs for the matched NLL and NLO+NNNLL distributions. As expected, the width of the NLO+NNNLL band, obtained varying the hard, soft and the factorization scale between 0.5 and 2 times their default value is significantly smaller than the band due to scale uncertainty in the (fixed order) NLO result.

We obtain the total cross section by integrating the invariant mass distribution. The numerical results are reported in Table 1 (left) with the scales and the PDFs uncertainties. In Table 1 (right) is shown the dependence of the total cross section from the sleptons masses. The resummation effect 

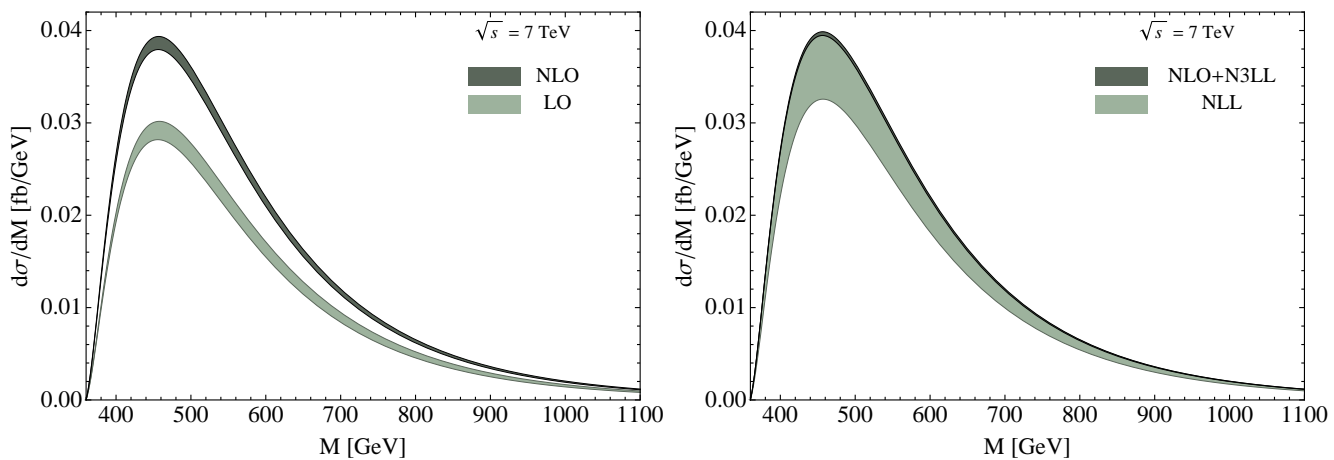

Figure 1: Invariant mass distribution for slepton-pair production. These plots are obtained for the following choice of the supersymmetric masses: $m_{\tilde{l}}=180 \mathrm{GeV}, m_{\tilde{q}}=600 \mathrm{GeV}$ and $m_{\tilde{g}}=750 \mathrm{GeV}$. In the left plots we show the fixed order result at full LO (light band) and NLO (dark band); in the right plot we show the matched result at NLL (light band) and NLO+NNNLL (dark band). The bands refer to the uncertainty associated with the variation of the scales.

\begin{tabular}{|l|c|}
\hline & LHC $(7 \mathrm{TeV})$ \\
\hline$\sigma_{\mathrm{LO}}$ & $8.01_{-0.36-0.34}^{+0.39+0.31}$ \\
$\sigma_{\mathrm{NLL}}$ & $9.59_{-0.64-0.37}^{+1.20+0.41}$ \\
$\sigma_{\mathrm{NLO}}$ & $10.56_{-0.22}^{+0.24}-0.43$ \\
$\sigma_{\mathrm{NNLL}}$ & $10.39_{-0.14}^{+0.10}+0.44$ \\
$\sigma_{\mathrm{NLO}+\mathrm{NNLL}}$ & $10.63_{-0.08}^{+0.08}+0.48$ \\
$\sigma_{\mathrm{N} 3} \mathrm{LL}$ & $10.56_{-0.09}^{+0.09}-0.45$ \\
$\sigma_{\mathrm{NLO}+\mathrm{N}^{3} \mathrm{LL}}$ & $10.81_{-0.06}^{+0.08}{ }_{-0.37}^{+0.48}$ \\
\hline
\end{tabular}

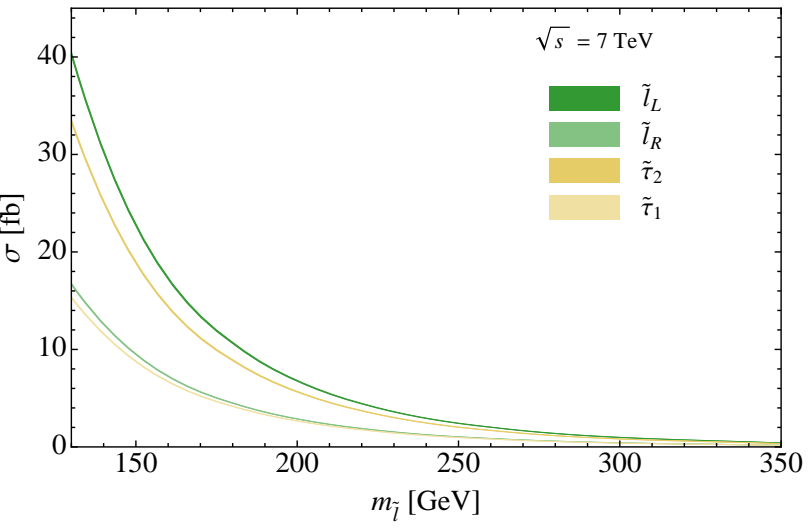

Table 1: Left: total cross sections in fb. The first error refers to the perturbative uncertainties associated with scale variations, the second to PDF uncertainties. The factorization scale $\mu_{f}$ is set equal to the invariant mass $M$. Right: Cross section for slepton-pair production as a function of the slepton mass for $m_{\tilde{g}}=750 \mathrm{GeV}$ and $m_{\tilde{q}}=600 \mathrm{GeV}$. This plot refers to our best prediction NLO+NNNLL.

for the LHC gives an additional contribution of the $3 \%$ on the total cross section respect to the NLO, bigger effects, of around $7 \%$, are found at the Tevatron [3].

In conclusion the threshold resummation has a relatively small effect on the cross sections, but it reduces significantly the scale dependence of the predictions.

\section{References}

[1] W. Beenakker, M. Klasen, M. Kramer, T. Plehn, M. Spira, P. M. Zerwas, Phys. Rev. Lett. 83 (1999) 3780-3783. [hep-ph/9906298].

[2] G. Bozzi, B. Fuks, M. Klasen, Nucl. Phys. B777 (2007) 157-181. [hep-ph/0701202].

[3] A. Broggio, M. Neubert and L. Vernazza, in preparation; T. Becher, M. Neubert, G. Xu, JHEP 0807 (2008) 030. [arXiv:0710.0680 [hep-ph]].

[4] A. D. Martin, W. J. Stirling, R. S. Thorne, G. Watt, Eur. Phys. J. C63 (2009) 189-285.

[hep-ph/0901.0002]. 